\title{
Molecular mapping and identification of quantitative trait loci for domestication traits in the field cress (Lepidium campestre L.) genome
}

\author{
Zeratsion Abera Desta ${ }^{1} \cdot$ Dirk-Jan de Koning $\mathbb{( D}^{2} \cdot$ Rodomiro Ortiz $^{1}$
}

Received: 7 September 2019 / Revised: 18 January 2020 / Accepted: 20 January 2020 / Published online: 19 February 2020

(c) The Author(s) 2020. This article is published with open access

\begin{abstract}
Lepidium campestre (L.) or field cress is a multifaceted oilseed plant, which is not yet domesticated. Moreover, the molecular and genetic mechanisms underlying the domestication traits of field cress remain largely elusive. The overarching goal of this study is to identify quantitative trait loci (QTL) that are fundamental for domestication of field cress. Mapping and dissecting quantitative trait variation may provide important insights into genomic trajectories underlying field cress domestication. We used 7624 single nucleotide polymorphism (SNP) markers for QTL mapping in $428 \mathrm{~F}_{2}$ interspecific hybrid individuals, while field phenotyping was conducted in $\mathrm{F}_{2: 3}$ segregating families. We applied multiple QTL mapping algorithms to detect and estimate the QTL effects for seven important domestication traits of field cress. Verification of pod shattering across sites revealed that the non-shattering lines declined drastically whereas the shattering lines increased sharply, possibly due to inbreeding followed by selection events. In total, 1461 of the 7624 SNP loci were mapped to eight linkage groups (LGs), spanning $571.9 \mathrm{cM}$ map length. We identified 27 QTL across all LGs of field cress genome, which captured medium to high heritability, implying that genomics-assisted selection could deliver domesticated lines in field cress breeding. The use of high throughput genotyping can accelerate the process of domestication in novel crop species. This is the first QTL mapping analysis in the field cress genome that may lay a foundational framework for positional or functional QTL cloning, introgression as well as genomics-assisted breeding in field cress domestication.
\end{abstract}

\section{Introduction}

A biennial self-pollinated plant, with relatively small genome size $(2 n=2 x=16$ and approximately $203.5 \mathrm{Mb})$ (Desta et al. 2019), field cress (Lepidium campestre L.) belongs to the Brassicaceae family. In addition to being a potential oilseed plant (Merker and Nilsson 1995; Nilsson et al. 1998), the winter hardy field cress can be: (i) undersown with a cereal as a catch crop (Merker et al. 2010) to recycle

Supplementary information The online version of this article (https:// doi.org/10.1038/s41437-020-0296-x) contains supplementary material, which is available to authorized users.

Zeratsion Abera Desta zeratsion.abera@slu.se

1 Department of Plant Breeding, Swedish University of Agricultural Sciences, Sundesvagen 10 Box 101, 23053 Alnarp, Sweden

2 Department of Animal Breeding and Genetics, Swedish University of Agricultural Sciences, Box 7023, 75007 Uppsala, Sweden vulnerable nutrients (e.g. nitrogen and phosphorous) from leaching (Ulén and Aronsson 2018); (ii) utilized as an ecofriendly solution to safeguard the environment and alleviate the negative consequences of climate change, such as nitrogen pollution and eutrophication; and (iii) used to domesticate new crops as a viable option to feed an ever-increasing global population, thus addressing the future food-security concerns.

Despite its potential uses, field cress remains undomesticated due to lack of research on the molecular and genetic mechanisms controlling its domestication traits. As majority of these traits are quantitative in nature (Doebley et al. 2006), mapping and analysis of quantitative trait loci (QTL) is the key starting point.

Crop domestication is a dynamic process that results into morphologically and physiologically divergent crops compared with their wild progenitors. Historically, domestication syndrome was done considering the properties of the wild variant of a crop (Hammer 1984), such as reduced seed shattering, free-threshing, large seeds or reduced seed dormancy, to obtain an edible variant of that crop species with various modified traits. Thus, crop domestication is a 

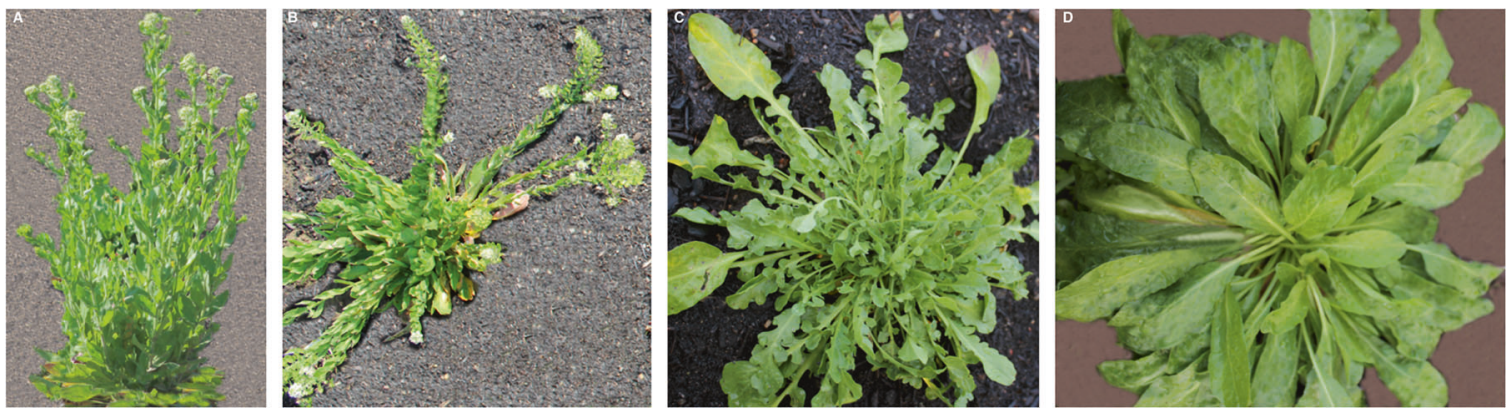

Fig. 1 Domestication traits of Lepidium. a Erect (up-right) type of plant architecture. b Prostrate type of plant architecture. c Indented (lobed) leaf morphology. d Non-indented (non-lobed) leaf morphology. Photo credit by Desta.

process of altering the morphological or physiological traits of underlying genes to achieve the intended end usage (Doebley et al. 2006). In addition to food and technological innovation, domestication can also be used for genome evolution and adaptive selection (Purugganan and Fuller 2009; Walsh and Lynch 2018).

Similar to other members of Brassicaceae (Gan et al. 2008, 2016; Jie et al. 2017), pod dehiscence (release of seeds from the mother plant) is a domestication trait resulting in yield loss in field cress cultivation. Many experimental studies have isolated and characterized the genes associated with seed shattering, e.g., in barley (Pourkheirandish et al. 2015), wheat (Simons et al. 2006), rice ( $\mathrm{Li}$ et al. 2006), sorghum (Lin et al. 2012), and tomato (Vrebalov et al. 2009). Therefore, cloning of genes attributed to pod shattering QTL is essential to accelerate the process of domestication and improve the yield.

Field cress has significant variations in traits, such as leaf morphology (e.g. leaf indentation) and plant architecture. However, these patterns (e.g. leaf shape, leaf arrangement) are not random but highly organized and systematically controlled variations (Juenger et al. 2005; Klingenberg 2002). The teosinte branch 1 (tb1) (Doebley et al. 1995; Doebley et al. 1997) and barren stalk 1 (bal) in maize (Gallavotti et al. 2004) and PROSTRATE GROWTH 1 (PROG1) in rice (Jin et al. 2008) are some of the welldefined genes controlling the architecture of plants. Thus, an extensive research on morphology-related traits of field cress will not only accelerate the domestication process, but also contribute to the understanding of plant growth and development across the members of Brassicaceae.

Due to their frequent and stable occurrence across the genomes, high-throughput sequencing platforms of single nucleotide polymorphism (SNP) discoveries have gained much attention and popularity (Brookes 1999; Shastry 2002). Notably, both the availability and distribution of SNPs across chromosomes lead to the establishment of ultrahigh density linkage maps (Vukosavljev et al. 2016). Linkage analysis can be used to determine how the patterns of parental genes paired with the marker loci (e.g. SNP loci) are co-inherited in the derived offspring population.

This study aims to identify the putative QTL regions pivotal in field cress domestication. It is the first QTL analysis for domestication traits using 7624 SNP markers in $428 \mathrm{~F}_{2}$ population of $L$. campestre. We identified major and minor QTL, whose functional validation may not only provide a venue to understand the process of domestication, but also illuminate the positional or functional QTL cloning, introgression of desirable QTL, as well as genomicsassisted breeding strategies in field cress domestication.

\section{Materials and methods}

\section{Plant material and phenotyping}

Two half-sib $F_{1}$ plants were obtained by crossing two separate field cresses as maternal parents with $L$. heterophyllum as a common pollen donor parent. A total of $428 \mathrm{~F}_{2}$ progenies were obtained by selfing the $F_{1}$ plants. Although field cress is biennial and $L$. heterophyllum is a perennial species, both plants are self-pollinating type. Field phenotyping was performed in the derived individuals of $\mathrm{F}_{2: 3}$ families to accommodate the trial of segregating populations with replications.

Seedlings were initially raised in a greenhouse and vernalized in a cold room for about six weeks. In 2014, the $F_{2: 3}$ individual lines were planted in field using three blocks with six replications. We recorded seven important agromorphological traits for domestication and yield improvement: plant height $(\mathrm{cm})$, inflorescence height $(\mathrm{cm})$, stem number, plant architecture, pod shattering, growth habit, and leaf morphology. Plant architecture was recorded based on the angle of the stem positions, i.e., erect plant architecture is upright position of stems (Fig. 1a), whereas the semi-erect plant architecture is an angle lower than erect but higher than semi-prostrate position. Prostrate position is the stems lying on the ground (Fig. 1b), while semi-prostrate is an angle positioned between semi-erect and prostrate architectures. 


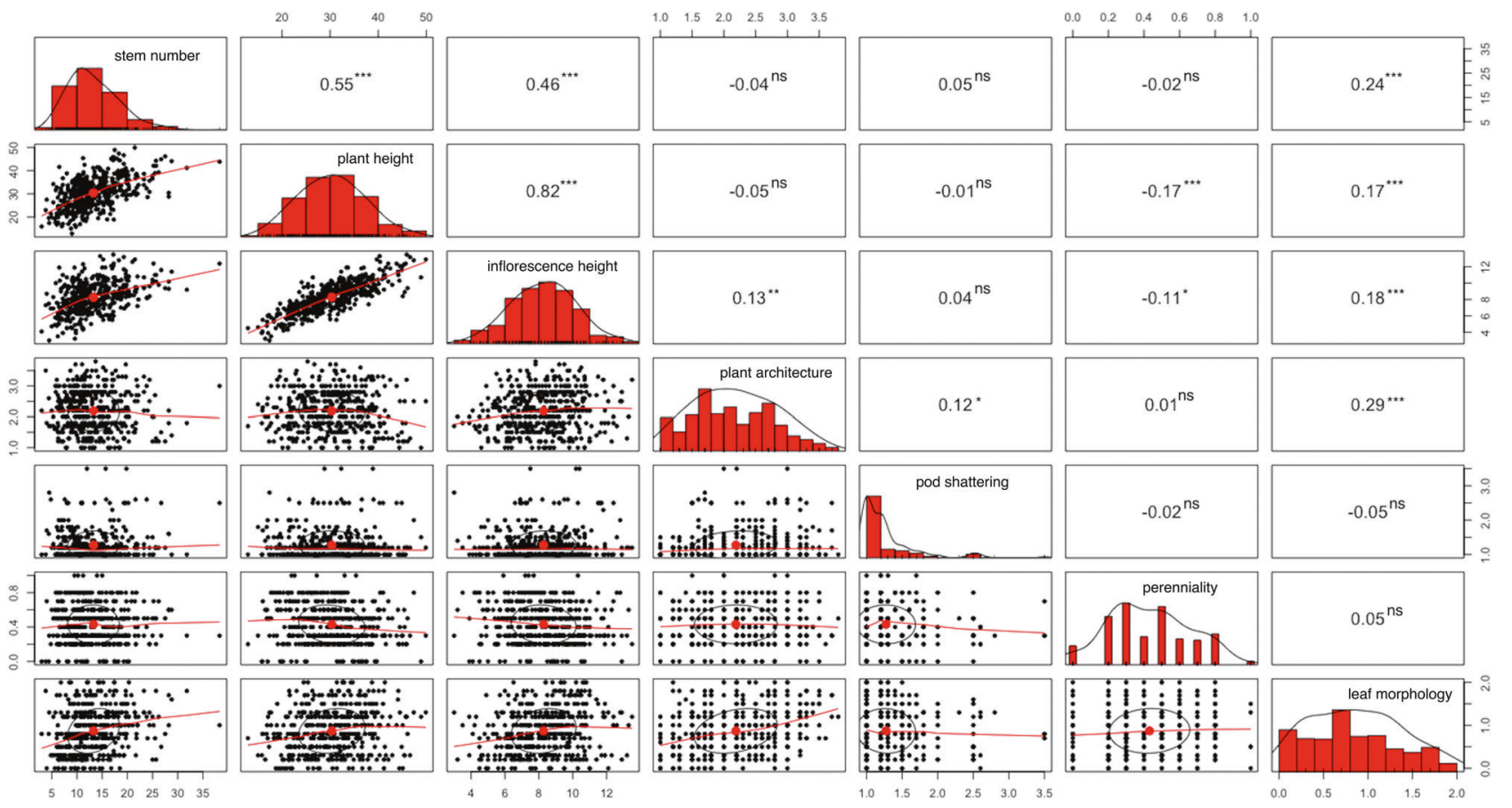

Fig. 2 Correlations ( $r$ ) (above diagonal) and scatter plot matrices (below diagonal) among domestication traits of field cress. Diagonal plots represent histogram distributions for domestication traits.
The ns indicates the $r$ value is statistically non-significant. The number of asterisk(s) show the level of significance $(* P<0.05),(* * P<0.01)$ and $(* * * P<0.001)$.
Leaf morphology was determined based on the extent of indentation or lobing, e.g., highly indented, medium indented, and non-indented leaves (see Fig. 1c, d). Plants were left in the field for an extended period of time (in 2015) to investigate the perenniality, and those which eventually survived till the next season were considered perennial. Pod shattering was evaluated as shattering (for sensitive genotype) and non-shattering (for reduced shattering) genotypes. Individuals retaining approximately $<75 \%$ and $\geq 75 \%$ seeds in the mother plants were recorded as shattering and non-shattering genotypes, respectively. To verify this, we further evaluated the $F_{4}$ progenies in 2015 at Alnarp (southern Sweden). Next, the selected individuals of $\mathrm{F}_{4}$ progenies were again phenotyped with $\mathrm{F}_{5}$ progenies in 2016 both at Alnarp and Uppsala (central Sweden).

\section{Phenotypic analysis}

Using the mixed models, variance components were estimated and significance tests were determined through loglikelihood ratio. To analyze the variance generated from the mixed models, we used the following matrix notation:

$Y=\boldsymbol{X} \boldsymbol{\beta}+\boldsymbol{Z} \boldsymbol{u}+\epsilon$,

where $Y$ is a known vector of phenotypic trait observations; $\boldsymbol{X}$ is the known design matrix of fixed predictors, relating observations in $Y$ to $\boldsymbol{\beta} ; \boldsymbol{\beta}$ is an unknown vector of fixed effects; $\boldsymbol{Z}$ is the known incidence matrix of random effects, relating the observations in $Y$ to $\boldsymbol{u} ; \boldsymbol{u}$ is an unknown vector of random effects, with a multivariate normal (MVN) distribution with mean vector 0 and a variance-covariance matrix denoted by $\boldsymbol{G}$, that is, $\boldsymbol{u} \sim N(\mathbf{0}, \boldsymbol{G})$; and $\in$ is an unknown vector of random residuals with an MVN distribution with mean vector 0 and a positive-definite variance-covariance matrix denoted by $\boldsymbol{R}$, that is, $\in \sim N(\mathbf{0}$, $\boldsymbol{R})$. Both $\boldsymbol{u}$ and $\in$ are independent. In our experiment, the genetic variance component $\left(\delta^{2} g\right)$ consists of the $\mathrm{F}_{2: 3}$ hybrid genotype effect, the interaction between the genotype and block effect, and the replication nested within a block effect, in which all of these components, including the residual variance $\left(\delta^{2} \in\right)$, were treated as random effects, whereas the overall mean was treated as fixed effect. The analyses for mixed models were performed using ASRemlR 3 (Butler et al. 2009).

Broad sense heritability $\left(H^{2}\right)$, estimated (Table 2$)$ as a ratio of genetic variance to the phenotypic variance (Falconer and Mackay 1996), includes both genetic and residual variance components. The correlations (r) between domestication traits (Fig. 2) were computed as given by Steel and Torrie (1980). Significance of each correlation was determined using a $t$-statistic test, after a $z$-score transformation of the correlation coefficient, to examine the hypothesis stating that the correlation is different from zero (i.e., different from nolinear association). We examined the coefficient of determination to estimate the amount of shared variation between traits with the help of ggm library 
(Marchetti 2006). Correlations between domestication traits were also implemented in R-3.6.1 (R Core Team 2018).

\section{DNA Isolation and genotyping}

DNA was isolated from young and fresh field cress leaves of the $\mathrm{F}_{2}$ progenies using cetyl trimethylammonium bromide (CTAB) protocol with some modifications in SaghaiMaroof et al. (1984) method. SNPs were developed from restricted amplified DNA (RAD) sequences sampled from the accessions of Lepidium (Lopes-Pinto et al. 2016). DNA samples of the $428 \mathrm{~F}_{2}$ individuals were sent to Edinburgh Genomics (http://genomics.ed.ac.uk) for genotyping using iSelect Illumina Infinium technology. The genotype calls and image files were processed using GenomeStudio (Illumina Inc. San Diego, CA, USA) to extract the data. Problematic SNP markers were identified by their poor clusterseparation values - that is lower Norm R and Norm Theta scores. These poor-quality SNPs were visually assessed and excluded, and 7624 SNPs were finally employed to genotype the $428 F_{2}$ individuals of $L$. campestre.

\section{Genetic linkage map construction}

This analysis included marker loci and individuals with less than 5\% missing values. After pre- and post-map quality control, 1461 of the 7624 SNP markers, in 428 individuals, were used for the final linkage map construction of field cress. Genetic map construction was carried out with JoinMap 4.1 (Van Ooijen 2006) using maximum likelihood mapping algorithm. The independent logarithm of the odds (LOD) parameters, ranging from 3 to 11 , were used to assign linked markers to their corresponding linkage groups (LGs). A linkage map was constructed for each LG using combinations of Gibbs sampling, simulated annealing, and spatial sampling. Linkage mapping function was computed using Haldane's mapping function (Haldane 1919), and LG maps were derived through MapChart 2.2 (Voorrips 2002). Synteny analysis data were accessed from previous research on L. campestre (Desta et al. 2019). Allele frequencies with one of the alleles, $\mathrm{p}$, minus 0.5 , were computed to determine the expected allele frequencies for both $\mathrm{F}_{2}$ population and candidate QTL.

\section{QTL map analysis}

Phenotypic data were initially checked to see whether they follow a normal distribution, which was determined by the probability $(P)$ values of the Shapiro-Wilk test for normality-an assumption of the analysis of variance (ANOVA). According to this test, both plant and inflorescence heights met the criteria of normality.

Phenotypic data of $\mathrm{F}_{2: 3}$, along with the SNP scores of the mapping population and linkage maps of field cress, were imported and analysed in MapQTL ${ }^{\circledR} 6$ (Van Ooijen 2009). We quantified the explained phenotypic variation $\left(R^{2}\right)$ for each trait, as well as the additive variance for plant height, to demonstrate the variation in domestication traits. The relative positions and effects of putative QTL on plant and inflorescence heights were analyzed with initial interval mapping (IM) followed by an automatic backward elimination of cofactors recognized in the IM. The loci detected in backward elimination were fitted again to use the multiple QTL mapping (MQM) method (Jansen and Stam 1994; Zeng 1994). MQM is a robust algorithm as it combines multiple regression analysis with IM (Jansen and Stam 1994) and fits the significant loci in the same or different chromosomes as a cofactor to increase the precision of QTL detection while minimizing the residual variations.

Non-parametric estimator Kruskal-Wallis (KW) ranksum test was applied to analyze the QTL for leaf morphology, plant architecture, pod shattering, perenniality, and stem number. The KW rank-test, equivalent to a one-way ANOVA, approximately follows the chi-square distribution $\left(\chi^{2}\right)$, including its degree of freedom, which assumes, under null hypothesis, that there is no effect on the segregating QTL (Van Ooijen 2009). KW test was initially employed with $P<0.005$ significant threshold level to examine the QTL-marker associations. This was followed by a further analysis of the significant loci from the KW test using MQM approach to locate and estimate the QTL effects.

LOD scores surpassing a genome-wide error rate of $5 \%$ $(P \leq 0.05)$, using 10,000 permutations (Churchill and Doerge 1994), were considered a significant association between SNP and trait loci. The mapping step size was $1 \mathrm{cM}$ with a maximum of 5 neighboring markers and 200 iterations. The support interval was established as a $95 \%$ support level or confident interval (CI) for two corresponding LOD drop-offs on either sides of the surrounding QTL peak. QTL with an overlapping support level were represented by the highest peak value in that CI. To ascertain the epistasis effects across multiple QTL candidates, we studied the interaction effect among candidate loci in R-3.6.1 ( $\mathrm{R}$ Core Team 2018).

\section{Results}

\section{Phenotypic evaluations}

\section{Correlation and shared variation between traits}

The correlation coefficients $(r)$ between measured phenotypic traits ranged from 0.01 to 0.82 (Fig. 2). Plant height had a statistically significant positive correlation $(r=0.82$; $P<0.001$ ) with inflorescence height (Fig. 2). Similarly, significant positive correlations were noticed, for example, 
Table 1 The proportion of variation $(\%)$ shared between phenotypic traits of Lepidium campestre.

\begin{tabular}{lccccccc}
\hline Traits & Arch. $^{\mathrm{a}}$ & Stem nr & Plant height & Infl. ht. $^{\mathrm{b}}$ & Pod sht. $^{\mathrm{c}}$ & Pern. $^{\mathrm{d}}$ & Lf mor. $^{\mathrm{e}}$ \\
\hline Arch. $^{\mathrm{a}}$ & - & 0.8 & 6.4 & 8.2 & 1.6 & 0.1 & 10.4 \\
Stem nr & 0.2 & - & 9.7 & 0.1 & 0.7 & 0.5 & 3.8 \\
Plant height & 0.3 & 30.1 & - & 60.1 & 0.4 & 2.9 & 0.6 \\
Infl. ht. $^{\mathrm{b}}$ & 1.7 & 21.1 & 66.6 & - & 0.3 & 0.4 & 0.2 \\
Pod sht. $^{\mathrm{c}}$ & 1.4 & 0.2 & 0.0 & 0.2 & - & 0.1 & 1.0 \\
Pern. $^{\mathrm{d}}$ & 0.0 & 0.0 & 2.9 & 1.1 & 0.0 & - & 0.5 \\
Leaf mor. $^{\mathrm{e}}$ & 8.6 & 5.9 & 3.0 & 3.1 & 0.3 & 0.3 & - \\
\hline
\end{tabular}

The variation accounted between phenotypic traits while all other five traits held constant. The variance contribution below diagonal indicates without accounting for the shared variations from the remaining five traits.

${ }^{a}$ Plant architecture.

${ }^{\mathrm{b}}$ Inflorescence height.

${ }^{\mathrm{c}}$ Pod shattering.

${ }^{\mathrm{d}}$ Perenniality.

${ }^{\mathrm{e}}$ Leaf morphology. between plant height and stem number $(r=0.55 ; P<0.001)$ and stem number and leaf morphology $(r=0.24, P<$ $0.001)$. The correlations of perenniality to all other traits were insignificant except for plant height $(r=-0.17 ; P<$ $0.001)$ and inflorescence height $(r=-0.11, P<0.05$; Fig. $2)$. We only found positively significant correlation $(r=$ $0.12 ; P<0.05)$ between pod shattering and plant architecture, whereas the remaining five traits revealed insignificant correlations with pod shattering.

The shared variations between traits were examined with (above diagonal; Table 1) and without keeping (below diagonal; Table 1) five of the seven domestication traits constant. A comparison of the variations showed that those contributing to below diagonal, for stem number versus plant height $(30.1 \%)$ and for stem number versus inflorescence height $(21.1 \%)$, were substantially higher than their shared variations above diagonal $(9.7$ and $0.1 \%$, respectively). These huge increments can be attributed to the additional variations from the remaining five domestication traits below diagonal (Table 1). On the other hand, in some traits, for instance, contribution of variation between plant architecture and inflorescence height below diagonal (1.7\%) was smaller than above diagonal ( $8.2 \%)$, possibly due to the antagonistic contributions of shared variations outweighing the positive contributions.

\section{Verification for pod shattering in field cress}

To test the performance of pod shattering, specifically its potential to increase the yield of field cress, we chose from the best performing non-shattering individuals of $\mathrm{F}_{2: 3}$ families, and further phenotyped these advanced progenies across years and sites. Shattering genotypes, obscured in non-shattering $\mathrm{F}_{2: 3}$ population, were revealed in the segregating individuals of $\mathrm{F}_{4}$ lines (Fig. $3 \mathrm{a}$ ). In the $\mathrm{F}_{5}$ lines, nonshattering genotypes declined a lot and even became nil in some lines (e.g. Hy25_12_5 and Hy25_23_1 lines at both sites; Fig. 3b, c). In subsequent generations, non-shattering genotypes declined dramatically whereas sensitive genotypes for pod shattering substantially increased (Fig. 3a-c).

\section{Genetic linkage mapping}

After quality control, 1461 of the 7624 SNP loci were mapped to eight LGs (Supplementary Fig. 1) with a total map length of $571.9 \mathrm{cM}$ (Table 2). LG7 had the largest lenght with $124.9 \mathrm{cM}$, whereas the highest number of markers were mapped to LG1 (409). Closer inspection of skewed loci incorporating $30 \%$ (440 of the 1461) of the SNP genotypes deviated $(P<0.05)$ from the Mendelian segregation ratios (Table 2). LG6 (173 loci) showed the highest segregation distortions followed by LG7 (86 loci), and then by LG3 (80 loci). No segregation distortion was detected in LG1, LG5, and LG8 (Table 2).

Across the eight LGs of L. campestre, 57\% (825 of 1461) of the SNP sequences were in conserved synteny while relating with $A$. thaliana chromosomes (Table 2). The highest and the lowest conserved synteny were identified in LG1 (260 loci) and LG5 (35 loci), respectively. Considering segregation distortion and homologies of loci, 255 of the 825 conserved synteny loci ( $\sim 31 \%$ of the conserved synteny loci or $\sim 17 \%$ of the SNP loci) showed pairing of both skewed segregation and conserved synteny (Table 2). Similar to the segregation distortion alone, LG6 showed the highest record (99 loci) when segregation distortions and conserved synteny loci were combined. 

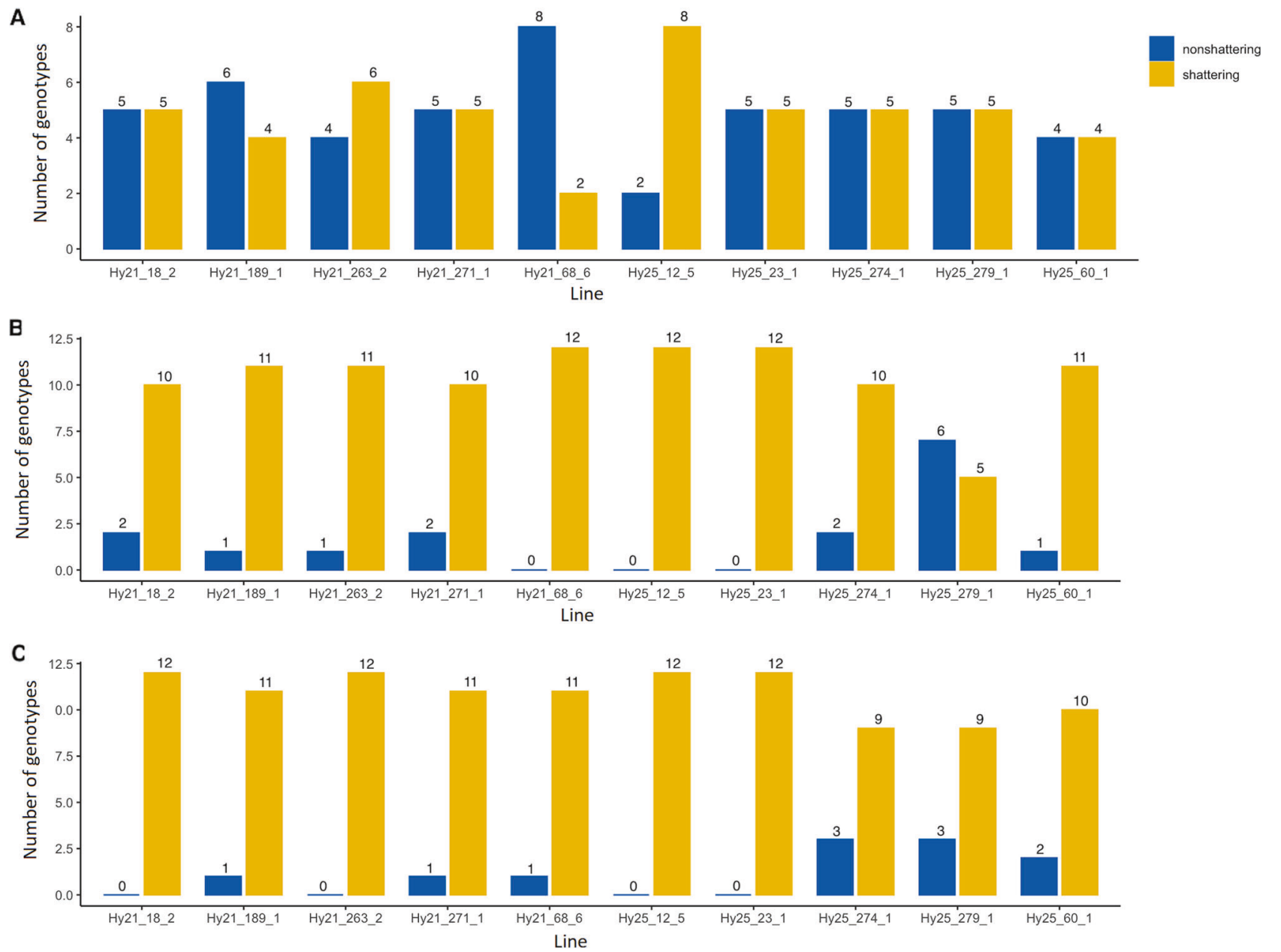

Fig. 3 Validation of the non-shattering lines of $F_{2: 3}$ population across years and sites. a The $\mathrm{F}_{4}$-generation with 10 replications in 2015 at one site that is, Alnarp, southern Sweden. b The $\mathrm{F}_{5}$-generation

with 12 replications in 2016 at Alnarp. c The $\mathrm{F}_{5}$-generation with 12 replications in 2016 at Uppsala (central Sweden).
Table 2 Number of SNP

markers, length of linkage groups (LGs) in cM, number of conserved syntenic and distorted loci in each LG of field cress.

\begin{tabular}{|c|c|c|c|c|c|c|c|c|c|c|c|}
\hline \multirow[t]{2}{*}{ LG } & \multirow[t]{2}{*}{ Locus } & \multirow[t]{2}{*}{ Size } & \multirow[t]{2}{*}{ Synteny } & \multirow[t]{2}{*}{ SegSyn $^{\mathrm{a}}$} & \multicolumn{7}{|c|}{ Segregation distortion } \\
\hline & & & & & A & B & $\mathrm{C}$ & $\mathrm{D}$ & $\mathrm{E}$ & $\mathrm{F}$ & Total \\
\hline 1 & 409 & 77.2 & 260 & - & - & - & - & - & - & - & - \\
\hline 2 & 209 & 74.2 & 101 & 44 & 21 & 9 & 13 & - & - & 1 & 44 \\
\hline 3 & 145 & 59.7 & 93 & 43 & 6 & 5 & 15 & 6 & 13 & 35 & 80 \\
\hline 4 & 180 & 75.0 & 73 & 30 & 15 & 1 & 2 & 2 & 10 & 27 & 57 \\
\hline 5 & 84 & 90.9 & 35 & - & - & - & - & - & - & - & - \\
\hline 6 & 207 & 46.1 & 122 & 99 & 55 & 39 & 32 & 17 & 22 & 8 & 173 \\
\hline 7 & 168 & 124.9 & 100 & 39 & 42 & 8 & 12 & 3 & 12 & 9 & 86 \\
\hline 8 & 59 & 23.9 & 41 & - & - & - & - & - & - & - & - \\
\hline Total & 1461 & 571.9 & 825 & 255 & 103 & 44 & 37 & 7 & 22 & 42 & 440 \\
\hline
\end{tabular}

${ }^{a}$ Segregation distortion and synteny, the level of distorted SNP loci is indicated by $\mathrm{A}=P<0.05, \mathrm{~B}=P<$ $0.01, \mathrm{C}=P<0.005, \mathrm{D}=P<0.001, \mathrm{E}=P<0.0005$ and $\mathrm{F}=P<0.0001$. 

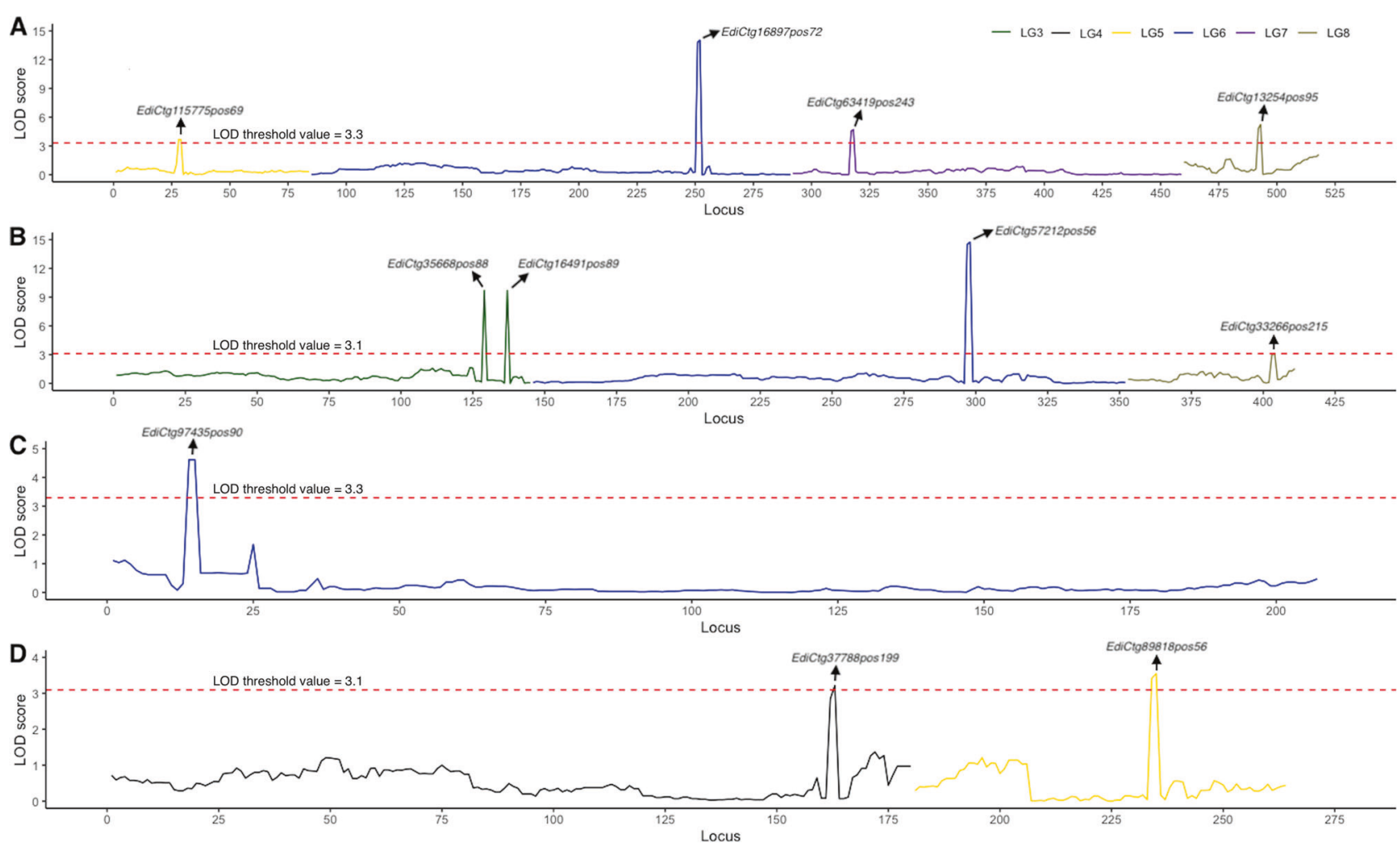

Fig. 4 The detection and estimation of QTL. a, b Plant and inflorescence heights. c Stem number. d Perenniality. The LOD scores are shown on the y-axis and the loci id numbers are ordered based on the

\section{QTL map analyses}

The most common segregations of favorable QTL alleles from field cress parents include increased plant and inflorescence heights, upright and semi-upright plant architectures, vigorous stem growth, and indehiscence (relative to L. heterophyllum). On the other hand, perenniality, semiprostrate and prostrate plant architectures, as well as highly indented or lobed leaf morphology (relative to field cress) are the possible favourable QTL segregations from the L. heterophyllum parent.

We identified a total of 27 QTL effects in seven domestication traits of field cress using MQM algorithms (Fig. 4a-d; Fig. 5a-c; Table 3). The support interval size varied between $0-0.1$ and $13.3-16.7 \mathrm{cM}$. The proportion of total explained phenotypic variation by QTL $\left(R^{2}\right)$ ranged from $4.9 \%$ (for stem number) to $39.8 \%$ (for leaf morphology). Consistent with the explained phenotypic variation in plant height $\left(R^{2}=12.4 \%\right.$; Table 3$)$, the highest peak in additive variance for plant height was obtained for LG6 (Fig. 6c). Small effects of QTL were identified across all LGs with all domestication traits, while LG1 (for both leaf morphology and pod shattering), LG2 (for plant architecture), and LG6 (for both plant and inflorescence heights) showed the major effect QTL regions in the field cress genomes. linkage map position. Arrows are used to indicate candidate loci for domestication traits. The red dashed lines are used to indicate the LOD threshold values.

Compared with Arabidopsis genome, 13 of the 27 field cress candidate QTL had conserved synteny (Supplementary Data S1), four of which had skewed segregations in addition to synteny. To investigate the distributions of the expected allele frequencies, we computed and visualized it in both $\mathrm{F}_{2}$ population (Fig. 6a) and candidate QTL (Fig. 6b). Except for one candidate, QTL associated with perenniality (0.22; Fig. 6b), the pattern was relatively either close or at the expected allele frequency.

Multiple QTL were detected for all domestication traits except in stem number. To unravel the epistasis effects, we analyzed the interaction effects among candidate QTL. Based on this analysis, we found significant $(P<0.001)$ interaction effects among candidates of pod shattering QTL (Supplementary Data S2). However, the remaining five domestication traits did not show any significant interaction effects among the candidate QTL. The broad-sense heritability $\left(H^{2}\right)$ of traits varied between $18.7 \%$ (for perenniality) and $87.9 \%$ (for pod shattering) (Table 3 ).

\section{Discussion}

A total of 27 QTL were estimated across all LGs in seven domestication traits (Fig. 4a-d; Fig. 5a-c; Table 3), 13 of which included significantly $(P<0.05)$ skewed segregations 

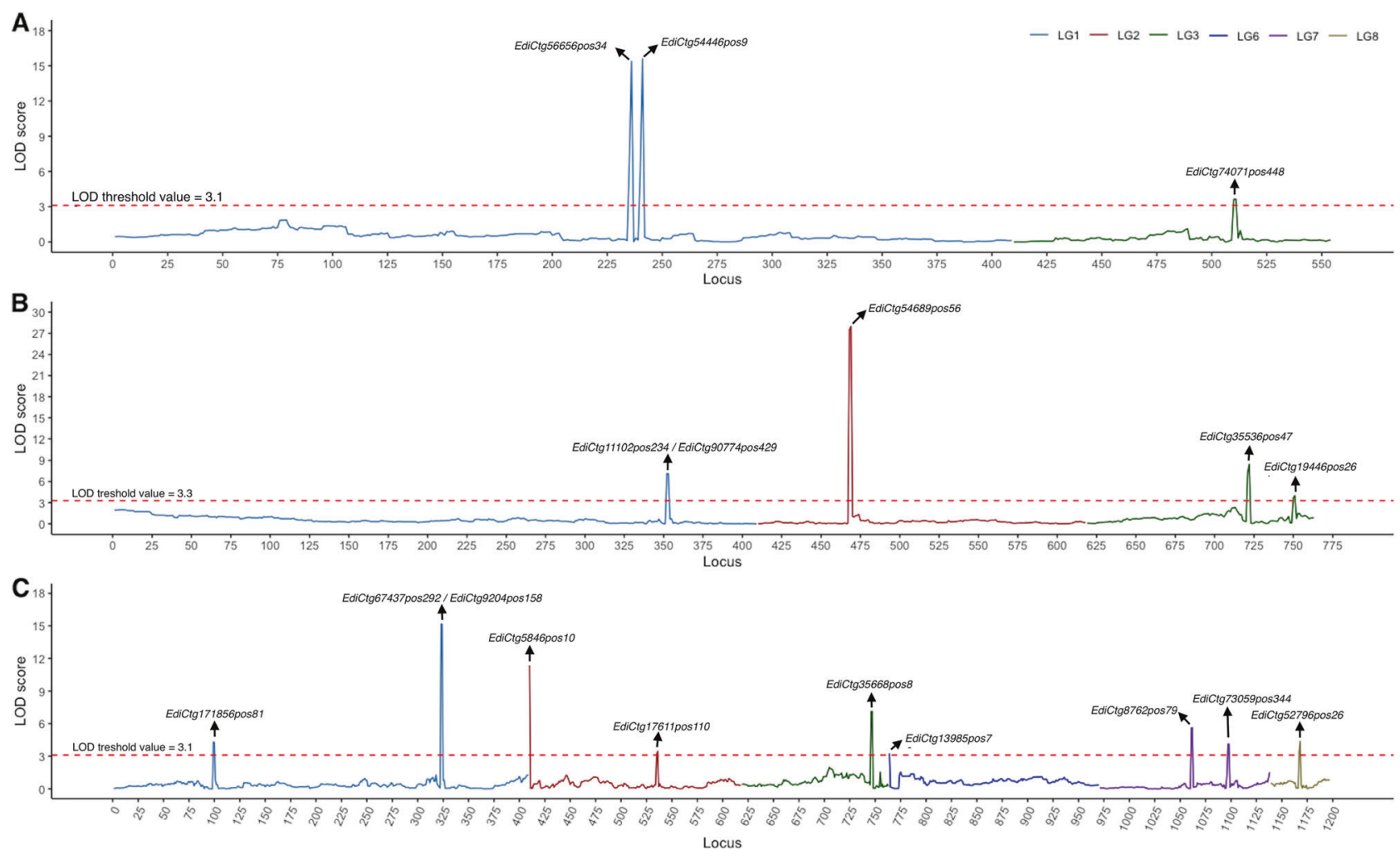

Fig. 5 The detection and estimation of QTL. a-c For pod shattering, plant architecture and leaf morphology, respectively. The LOD scores are shown on the y-axis and the loci id numbers are ordered based on the linkage map position. Arrows are used to indicate candidate loci for domestication traits. The red dashed lines are used to indicate the LOD threshold values.
(Supplementary Data S1). In linkage analysis, meticulous incorporation of skewed loci could have numerous biological benefits, such as to (i) attain good resolution of genetic maps (Zuo et al. 2019); (ii) find candidate QTL that are associated with traits of interest (Xu 2008); and (iii) gain access to conserved synteny compared with a reference genome (Table 2). In this study, excluding the distorted loci could have led to losses not only in 255 conserved synteny (Table 2), but also in 13 of the 27 candidate QTL (Supplementary Data S1).

Six major effect QTL (for pod shattering, leaf morphology, and plant inflorescence heights) were identified across three LGs (LG1, LG2, and LG6) of L. campetsre (Fig. 4a, b; Fig. 5a-c; Table 3). Large effect QTL are often stable across multiple environments (Dixit et al. 2014; Kato et al. 2014), and practically feasible for map-based or positional cloning of underlying genes (Mackay 2009). A major effect locus study was used to effectively position the candidate gene underlying the plant height QTL in maize (Teng et al. 2013). Thus, major effect QTL identified in this study may be key to positional or functional cloning of candidate genes in field cress improvement.

Plant architecture and plant height are interrelated traits that affect the yield of plants. Tall rapeseed plants, for example, are found to be prone to lodging, thereby affecting the seed yield at harvest (Mei et al. 2009). Plant architecture is a key feature that distinguishes a biennial field cress from a perennial $L$. heterophyllum. Erect and semi-erect stem positions are the usual features of field cress, while prostrate or semi-prostrate positions (see material and methods sections for their descriptions) are the typical plant architectures of L. heterophyllum. Stem lodging is one of the major challenges that a domesticated field cress must overcome.

Characterization of candidate domestication genes responsible for plant architecture was reported in crop species. The teosinte branch 1 ( $t b 1)$ that represses lateral branching in maize (Doebley et al. 1995, 1997) also has a pleiotropic effect on various plant architecture-related traits (Clark et al. 2006). The deactivation of the PROSTRATE GROWTH 1 (PROG1) gene that leads the prostrate growth toward erect position improves yield in rice (Jin et al. 2008; Tan et al. 2008). The identification of deletion genes associated with Rice Plant Architecture Domestication (RPAD) locus in both African and Asian rice indicates a transfer site of the prostrate resulting in changing the low-yield wild rice to erect and high-yield domesticated rice (Wu et al. 2018). In addition, inactivating the large effect QTL Tiller Angle Control 1 (TAC1) transfers the non-compact architecture to compact and erect tillers in rice (Yu et al. 2007). Together, 
Table 3 Quantitative trait loci (QTL) for seven domestication traits.

\begin{tabular}{|c|c|c|c|c|c|c|c|c|}
\hline Trait & Locus & LG & LOD & Position (cM) & Confidence interval (cM) & $R^{2}(\%)$ & Total $R^{2}$ & $H^{2}$ \\
\hline \multirow[t]{4}{*}{ Plant height } & EdiCtg115775pos69 & 5 & 3.7 & 66.2 & $66.2-67.2$ & 3.1 & \multirow[t]{4}{*}{23.8} & \multirow[t]{4}{*}{47.9} \\
\hline & EdiCtg16897pos72 & 6 & 14.0 & 39.7 & $39.1-39.8$ & 12.4 & & \\
\hline & EdiCtg63419pos243 & 7 & 4.7 & 3.3 & $2.6-3.5$ & 3.9 & & \\
\hline & EdiCtg13254pos95 & 8 & 5.3 & 17.2 & $16.8-17.3$ & 4.4 & & \\
\hline \multirow[t]{4}{*}{ Inflorescence height } & EdiCtg35668pos88 & 3 & 9.7 & 49.7 & $49.7-50.2$ & 8.1 & \multirow[t]{4}{*}{26.3} & \multirow[t]{4}{*}{53.7} \\
\hline & EdiCtg16491pos89 & 3 & 9.7 & 52.0 & $51.9-52.1$ & 3.1 & & \\
\hline & EdiCtg57212pos56 & 6 & 14.8 & 36.6 & $36.3-36.8$ & 12.6 & & \\
\hline & EdiCtg33266pos215 & 8 & 3.1 & 22.6 & $22.2-23.0$ & 2.5 & & \\
\hline Stem number & EdiCtg97435pos 90 & 6 & 6.6 & 3.5 & $3.5-4.7$ & 4.9 & 4.9 & 37.0 \\
\hline \multirow[t]{3}{*}{ Pod shattering } & EdiCtg54446pos 90 & 1 & 15.4 & 41.2 & $40.7-41.4$ & 14.8 & \multirow[t]{3}{*}{36.8} & \multirow[t]{3}{*}{87.9} \\
\hline & EdiCtg56656pos347 & 1 & 15.3 & 42.1 & $42.0-42.6$ & 15.1 & & \\
\hline & EdiCtg74071pos 448 & 3 & 3.6 & 39.6 & $39.4-40.3$ & 3.3 & & \\
\hline \multirow[t]{2}{*}{ Perenniality } & EdiCtg37788pos 199 & 4 & 3.2 & 71.3 & $70.7-71.4$ & 3.3 & \multirow[t]{2}{*}{6.9} & \multirow[t]{2}{*}{18.7} \\
\hline & EdiCtg89818pos56 & 5 & 3.6 & 81.0 & $80.2-81.2$ & 3.6 & & \\
\hline \multirow[t]{4}{*}{ Plant architecture } & EdiCtg11102pos234/EdiCtg90774pos429 & 1 & 7.1 & 66.4 & $66.3-66.5$ & 4.1 & \multirow[t]{4}{*}{29.1} & \multirow[t]{4}{*}{87.0} \\
\hline & EdiCtg54689pos56 & 2 & 28.0 & 16.4 & $15.5-16.6$ & 17.9 & & \\
\hline & EdiCtg35536pos477 & 3 & 8.5 & 41.2 & $39.6-42.2$ & 4.9 & & \\
\hline & EdiCtg19446pos264 & 3 & 4.0 & 50.9 & $50.2-51.0$ & 2.2 & & \\
\hline \multirow[t]{9}{*}{ Leaf morphology } & EdiCtg171856pos81 & 1 & 4.3 & 15.0 & $14.9-15.2$ & 2.8 & \multirow[t]{9}{*}{39.8} & \multirow[t]{9}{*}{69.2} \\
\hline & EdiCtg67437pos292/EdiCtg9204pos158 & 1 & 15.2 & 61.0 & $60.8-61.1$ & 10.7 & & \\
\hline & EdiCtg5846pos 100 & 2 & 11.4 & 0.0 & $0.0-0.1$ & 7.8 & & \\
\hline & EdiCtg17611pos110 & 2 & 3.5 & 48.3 & $47.4-48.4$ & 2.3 & & \\
\hline & EdiCtg35668pos88* & 3 & 7.1 & 49.7 & $49.6-50.2$ & 4.8 & & \\
\hline & EdiCtg13985pos76 & 6 & 3.3 & 0.0 & $0.0-0.8$ & 2.1 & & \\
\hline & EdiCtg8762pos 79 & 7 & 5.6 & 13.4 & $12.8-13.5$ & 3.7 & & \\
\hline & EdiCtg73059pos344 & 7 & 4.1 & 105.3 & $104.1-106.3$ & 2.7 & & \\
\hline & EdiCtg52796pos267 & 8 & 4.4 & 16.1 & $13.3-16.7$ & 2.9 & & \\
\hline
\end{tabular}

It represents the location as described with each linkage group (LG), LOD score value, the proportion of phenotypic variance explained $\left(R^{2}, \%\right)$ by individual (each candidate locus) and joint QTL (total $R^{2}$ per trait), as well as the broad sense heritability $\left(H^{2}\right)$ of each trait.

${ }^{\mathrm{a}} \mathrm{A}$ locus expressed in both inflorescence height and leaf morphology.

these results indicate that genes that regulate the QTL of plant architecture-related traits can not only be used to transfer the prostrate to erect position, but can also improve the potential yield of plants.

Pod dehiscence is another important domestication trait that affects the yield of several plant species (Funatsuki et al. 2014; Gan et al. 2008; Jie et al. 2017; Mohammed et al. 2014). Although field cress and L. heterophyllum both undergo severe pod shattering, the former has relatively lower shattering than the latter. A loss of $\sim 0.5 \mathrm{t} \mathrm{ha}^{-1}$ is reported due to pod shattering in canola (Brassica juncea) unless early harvest is practised (Gan et al. 2016). With the identification of double mutant Shatterproof-1 and Shatterproof-2 genes, indehiscent pods were obtained in $A$. thaliana (Liljegren et al. 2000). Similar results of reduced shattering have been reported in rice (Konishi et al. 2006; Li et al. 2006; Lin et al. 2007; Wu et al. 2017), and soybean
(Dong et al. 2014; Funatsuki et al. 2014). Collectively, these results suggest that finding a reliable non-shattering QTL followed by isolation and characterization of underlying genes will substantially accelerate the domestication and yield of novel plants—-such as field cress.

In dicot species (e.g. members of Brassicaceae family), formation of the abscission layer is introduced to induce pod shattering near or after maturity (Dong and Wang 2015; Patterson 2001). Research in Arabidopsis revealed that comprehensive regulatory networks (e.g. the MADS box transcription factor) and tissues (Kay et al. 2013; Liljegren et al. 2000; Liljegren et al. 2004), as well as phytohormones (Arnaud et al. 2010; Cecchetti et al. 2008; Marsch-Martínez et al. 2012; van Gelderen et al. 2016) are the hallmarks to regulate the process of pod shattering. Given the close phylogenetic position for both field cress and Arabidopsis, coupled with the glimpse of high-throughput sequencing, 


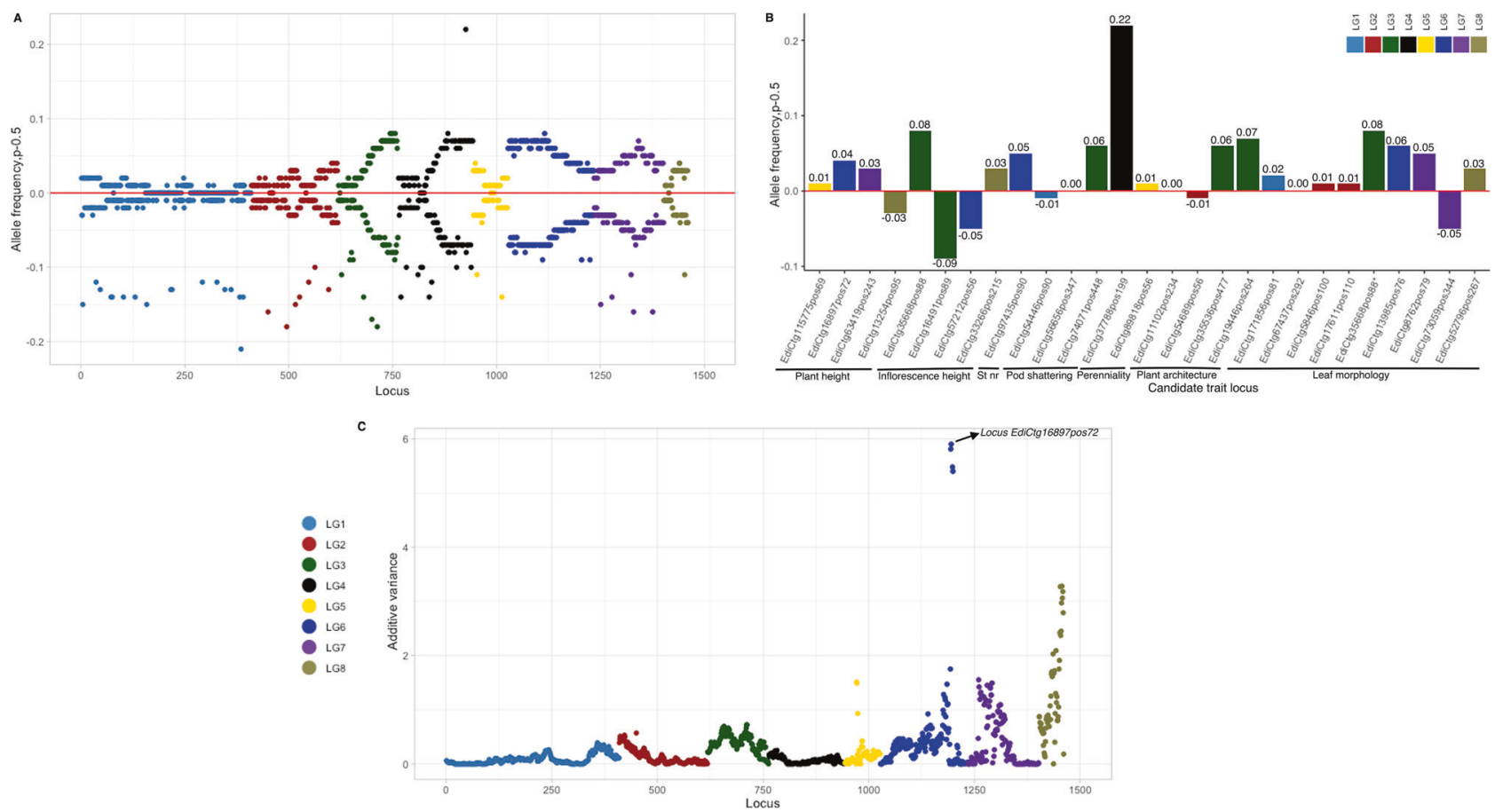

Fig. 6 Allele frequency with one of the alleles, $p,-0.5$ in both F2 population and QTL candidates, and the additive variance of plant height. a, b The frequency of $p-0.5$ at each locus in $F_{2}$ population, as well as at each candidate trait locus. Values above and below the abscissa represents positive and negative values, respectively. The patterns of the expected allele frequencies of the $F_{2}$ and candidate QTL were visualised compared with the expected allele at abscissa. The red line at the abscissa indicates the expected frequency

the above results can be translated into field cress to understand the physiological and molecular trajectories responsible for pod shattering.

Our validation of phenotyping across environments and years demonstrated that as the number of generations increased, the number of non-shattering individuals decreased drastically, and the shattering genotypes sharply increased (Fig. 3a-c). The decline of non-shattering genotypes across generation was probably driven by the phenomenon of inbreeding followed by selection (Bosse et al. 2019; Jamieson et al. 2008). Inbreeding is, however, unavoidable biological event, which could be used to generate pure lines as well as to increase the genetic similarity among relatives (Walsh and Lynch 2018). Genetic drift may also eliminate favourable alleles while fixing the disadvantageous alleles (Gaut et al. 2018; Wright et al. 2008). Thus, simultaneous inbreeding and selection in a small effective population size may also dramatically eliminate the useful mutation from a population.

Considering the agro-ecosystem, perennial plants have manifold benefits, such as eliminating the need for soil tillage, efficient utilization of natural resources (King and Blesh 2018), improving soil-carbon composition, and of allele. The domestication trait names are indicated below the dashed lines and the st $\mathrm{nr}$ is abbreviated for stem number. c The additive variance of plant height represented at each locus. The arrow indicating the candidate locus associated with the highest peak of additive variance at linkage group 6 (LG6) and this locus also explained $12.4 \%$ of the total phenotypic variation in plant height (Table 3 ). The loci id numbers along the $\mathrm{x}$-axis in $\mathbf{a}, \mathbf{c}$ are ordered based on the linkage map position.

minimizing soil degradation (DeHaan and Van Tassel 2014). On the other hand, perenniality also impedes agricultural tillage and harvesting machines when intercropped with annual cereals. Furthermore, intercropping perennially developed L. campestre (as a consequence of crossbreeding with $L$. heterophyllum) in arable lands could have a reverse effect on soil nitrogen, accelerating nutrient leaching that leads to environmental contamination such as nitrogen pollution (Desta et al. 2019; Kanter et al. 2019). Field cress can minimize nitrogen or phosphorous leaching if it is planted in spring (Ulén and Aronsson 2018). However, the authors also indicated that nutrient leaching could be intensified due to over-mineralization if field cress is inter-planted in autumn. Hence, in crop husbandry, caution is warranted to interpret not only the influences with perenniality but also the inter-sowing season of field cress.

Similar to other weed species (Ikram et al. 2014), field cress also has a severe problem of seed dormancy. We have noticed that some accessions of field cress can remain without germination for an extended period in the soil unless seeds are treated with chemicals-such as gibberellic acid (GA). Recent research in soybean (Jang et al. 2015; Sun et al. 2015; Wang et al. 2018), rice (Sugimoto et al. 
2010), Medicago truncatula (Chai et al. 2016), and barley (Sato et al. 2016) sheds light on mechanisms of regulating seed dormancy in plants. In addition to finding the naturally existing ecotypes, further efforts are needed in using mutation-based screening for reduced dormancy to investigate seed dormancy in field cress.

The environment is one of the vital components that determines the growth and development of plants. To meet the temperature requirement, we vernalised seedlings of field cress before transplanting them in the field; however, we noticed some variability in flowering. Hence, further research on photoperiod regulation and transition to flower initiation, as well as genes that control vernalisation, is central to facilitate field cress domestication.

In conclusion, to the best of our knowledge, this is the first study focusing on the estimation of QTL effects on seven agro-morphological traits that are key to facilitate field cress domestication. The correlation among these traits may not only assist in selection, but also contribute to examine pleiotropy effects when multiple QTL are involved. We found relatively small support sizes (ranging $0.1-3.4 \mathrm{cM}$ ) in the analyzed QTL regions, mimicking fine mapping in which SNPs are interlinked with each other to precisely locate the QTL positions. We found six large effect QTL, whereas the remaining 21 candidate QTL showed small effect QTL. Majority of the identified QTL captured moderate to high heritability, roughly $>40 \%$, demonstrating that genomics-assisted selection could deliver improved lines within a few generations of field cress breeding. The use of high-throughput genotyping platform (e.g. SNPs discovered from high-throughput sequencing) can immensely accelerate the process of domestication in novel crop species. The QTL results presented here may eventually provide avenue for map-based or mutation-based (e.g. CRISPR-induced mutations) QTL cloning; introgression of favourable genes in recipient parents; and genomics-assisted breeding approaches (e.g. genomic selection) in field cress domestication.

\section{Perspective}

While the identified QTL in this study could provide valuable contributions in QTL cloning and genomic-assisted breeding, future efforts are remarkably important in getting complete reference genome of field cress. This refence sequence will broaden and advance the in-depth understanding not only on how to explore the existing variation, but also on expanding alternatives of genomic tools in field cress domestication and improvement. Equally important, the QTL hotspot regions combined with fully annotated genome sequences are plausible to systematically correlate and answer the complicated questions in domestication genomics.

\section{Data archiving}

Genotype and phenotype data available from the Dryad Digital Repository: https://doi.org/10.5061/dryad.1jwstqjir4 (and via the direct link https://datadryad.org/stash/share/ qtSyvROqbnr0aKOGZ8KpRbzpiUAprOpNkzizZkI47Qc).

Acknowledgements This research was supported by the "Mistra Biotech" project, a research programme financed by the Swedish foundation for strategic environmental research (MISTRA) and Swedish University of Agricultural Sciences (SLU). We thank Mulatu Geleta (SLU) for providing the hybrids of Lepidium. We thank Johan W. Ooijen (Kyazma, Netherlands) for the advises in QTL mapping. We also thank Anna Zborowska and Cecilia Gustafson for their help with DNA extraction and sending the DNA samples to Scotland. We want to thank Vehbo Hot, Nils-Ove Bertholdsson, Erik Rasmusson, Alexandra Nikolic, Lucy Clark, Ewa Magnuski and Jannie Hagman for assisting the field planting of field cress. We acknowledge Richard Talbot (Edinburgh Genomics Institute) for introducing the methodology related to Illumina iSelect genotyping. We thank Jim Holland for comments on the variance component analysis.

Author contributions ZAD recorded and analysed the data, and wrote the manuscript. DJK and RO edited and revised the manuscript.

\section{Compliance with ethical standards}

Conflict of interest The authors declare that they have no conflict of interest.

Publisher's note Springer Nature remains neutral with regard to jurisdictional claims in published maps and institutional affiliations.

Open Access This article is licensed under a Creative Commons Attribution 4.0 International License, which permits use, sharing, adaptation, distribution and reproduction in any medium or format, as long as you give appropriate credit to the original author(s) and the source, provide a link to the Creative Commons licence, and indicate if changes were made. The images or other third party material in this article are included in the article's Creative Commons licence, unless indicated otherwise in a credit line to the material. If material is not included in the article's Creative Commons licence and your intended use is not permitted by statutory regulation or exceeds the permitted use, you will need to obtain permission directly from the copyright holder. To view a copy of this licence, visit http://creativecommons. org/licenses/by/4.0/.

\section{References}

Arnaud N, Girin T, Sorefan K, Fuentes S, Wood TA, Lawrenson T et al. (2010) Gibberellins control fruit patterning in Arabidopsis thaliana. Genes Dev 24(19):2127-2132

Bosse M, Megens HJ, Derks MF, de Cara ÁM, Groenen MA (2019) Deleterious alleles in the context of domestication, inbreeding, and selection. Evolut Appl 12(1):6-17

Brookes AJ (1999) The essence of SNPs. Gene 234(2):177-186

Butler D, Cullis B, Gilmour A, Gogel BA-R (2009) Reference manual, release 3.0. Queensland Department of Primary Industries and Fisheries, Brisbane 
Cecchetti V, Altamura MM, Falasca G, Costantino P, Cardarelli M (2008) Auxin regulates Arabidopsis anther dehiscence, pollen maturation, and filament elongation. Plant Cell 20(7):1760-1774

Chai M, Zhou C, Molina I, Fu C, Nakashima J, Li G et al. (2016) A class II KNOX gene, KNOX4, controls seed physical dormancy. Proc Natl Acad Sci 113(25):6997-7002

Churchill GA, Doerge RW (1994) Empirical threshold values for quantitative trait mapping. Genetics 138(3):963-971

Clark RM, Wagler TN, Quijada P, Doebley J (2006) A distant upstream enhancer at the maize domestication gene $t b l$ has pleiotropic effects on plant and inflorescent architecture. Nat Genet 38(5):594

DeHaan LR, Van Tassel DL (2014) Useful insights from evolutionary biology for developing perennial grain crops. Am J Bot 101 (10): 1801-1819

Desta ZA, Kolano B, Shamim Z, Armstrong SJ, Rewers M, Sliwinska $E$ et al. (2019) Field cress genome mapping: integrating linkage and comparative maps with cytogenetic analysis for rDNA carrying chromosomes. Sci Rep 9(1):17028

Dixit S, Singh A, Cruz MTS, Maturan PT, Amante M, Kumar A (2014) Multiple major QTL lead to stable yield performance of rice cultivars across varying drought intensities. BMC Genet 15(1):16

Doebley J, Stec A, Gustus C (1995) Teosinte branchedl and the origin of maize: evidence for epistasis and the evolution of dominance. Genetics 141(1):333-346

Doebley J, Stec A, Hubbard L (1997) The evolution of apical dominance in maize. Nature 386(6624):485-488

Doebley JF, Gaut BS, Smith BD (2006) The molecular genetics of crop domestication. Cell 127(7):1309-1321

Dong Y, Wang Y-Z (2015) Seed shattering: from models to crops. Front Plant Sci 6:476

Dong Y, Yang X, Liu J, Wang B-H, Liu B-L, Wang Y-Z (2014) Pod shattering resistance associated with domestication is mediated by a NAC gene in soybean. Nat Commun 5:3352

Falconer DS, Mackay TFC (1996) An introduction to quantitative genetics, 4th edn. Prentice Hall, London

Funatsuki H, Suzuki M, Hirose A, Inaba H, Yamada T, Hajika M et al. (2014) Molecular basis of a shattering resistance boosting global dissemination of soybean. Proc Natl Acad Sci 111(50):17797-17802

Gallavotti A, Zhao Q, Kyozuka J, Meeley RB, Ritter MK, Doebley JF et al. (2004) The role of barren stalk1 in the architecture of maize. Nature 432(7017):630

Gan Y, Blackshaw RE, May WE, Vera C, Johnson EN (2016) Yield stability and seed shattering characteristics of Brassica juncea canola in the Northern Great Plains. Crop Sci 56(3):1296-1305

Gan Y, Malhi S, Brandt S, McDonald C (2008) Assessment of seed shattering resistance and yield loss in five oilseed crops. Can J Plant Sci 88(1):267-270

Gaut BS, Seymour DK, Liu Q, Zhou Y (2018) Demography and its effects on genomic variation in crop domestication. Nat Plants 4 (8):512-520

Haldane J (1919) The combination of linkage values and the calculation of distances between the loci of linked factors. J Genet 8 (29):299-309

Hammer K (1984) Das domestikationssyndrom. Die Kulturpflanze 32 (1): $11-34$

Ikram R, Tanveer A, Ata Z, Saqib M (2014) Dormancy studies on Euphorbia dracunculoides and Astragalus spp.: major weeds of arid areas. Planta Daninha 32(4):747-753

Jamieson IG, Grueber CE, Waters JM, Gleeson DM (2008) Managing genetic diversity in threatened populations: a New Zealand perspective. New Zealand J Ecol 32:130-137

Jang S-J, Sato M, Sato K, Jitsuyama Y, Fujino K, Mori H et al. (2015) A single-nucleotide polymorphism in an endo-1, 4- $\beta$-glucanase gene controls seed coat permeability in soybean. PLoS ONE 10 (6):e0128527
Jansen RC, Stam P (1994) High resolution of quantitative traits into multiple loci via interval mapping. Genetics 136(4):1447-1455

Jie K, Li X, Yang Y, Zhou G (2017) Effects of paclobutrazol on biomass production in relation to resistance to lodging and pod shattering in Brassica napus L. J Integr Agric 16(11):2470-2481

Jin J, Huang W, Gao J-P, Yang J, Shi M, Zhu M-Z et al. (2008) Genetic control of rice plant architecture under domestication. Nat Genet 40(11):1365-1369

Juenger T, Pérez-Pérez JM, Bernal S, Micol JL(2005) Quantitative trait loci mapping of floral and leaf morphology traits in Arabidopsis thaliana: evidence for modular genetic architecture. Evol Dev 7(3):259-271

Kanter DR, Bartolini F, Kugelberg S, Leip A, Oenema O, Uwizeye A (2019) Nitrogen pollution policy beyond the farm. Nat Food $1: 27-32$

Kato S, Sayama T, Fujii K, Yumoto S, Kono Y, Hwang T-Y et al. (2014) A major and stable QTL associated with seed weight in soybean across multiple environments and genetic backgrounds. Theor Appl Genet 127(6):1365-1374

Kay P, Groszmann M, Ross J, Parish R, Swain S (2013) Modifications of a conserved regulatory network involving INDEHISCENT controls multiple aspects of reproductive tissue development in Arabidopsis. N Phytologist 197(1):73-87

King AE, Blesh J (2018) Crop rotations for increased soil carbon: perenniality as a guiding principle. Ecol Appl 28(1):249-261

Klingenberg CP (2002) Morphometrics and the role of the phenotype in studies of the evolution of developmental mechanisms. Gene 287(1-2):3-10

Konishi S, Izawa T, Lin SY, Ebana K, Fukuta Y, Sasaki T et al. (2006) An SNP caused loss of seed shattering during rice domestication. Science 312(5778):1392-1396

Li C, Zhou A, Sang T (2006) Rice domestication by reducing shattering. Science 311(5769):1936-1939

Liljegren SJ, Ditta GS, Eshed Y, Savidge B, Bowman JL, Yanofsky MF (2000) SHATTERPROOF MADS-box genes control seed dispersal in Arabidopsis. Nature 404(6779):766-770

Liljegren SJ, Roeder AH, Kempin SA, Gremski K, Østergaard L, Guimil S et al. (2004) Control of fruit patterning in Arabidopsis by INDEHISCENT. Cell 116(6):843-853

Lin Z, Griffith ME, Li X, Zhu Z, Tan L, Fu Y et al. (2007) Origin of seed shattering in rice (Oryza sativa L.). Planta 226(1):11-20

Lin Z, Li X, Shannon LM, Yeh C-T, Wang ML, Bai G et al. (2012) Parallel domestication of the Shatteringl genes in cereals. Nat Genet 44(6):720-724

Lopes-Pinto F, Vanhala T, Geleta M, Risse J, Nichols J, Karim Gharbi $\mathrm{K}$ et al. (2016) RAD sequencing of diverse accessions of Lepidium campestre, a target species for domestication as a novel oil crop. In: Plant and animal genome conference XXIV, plant and animal genome, San Diego, CA, USA. https://pag.confex.com/pa g/xxiv/webprogram/Paper19403.html

Mackay TF (2009) Q\&A: genetic analysis of quantitative traits. J Biol $8(3): 23$

Marchetti GM (2006) Independencies induced from a graphical Markov model after marginalization and conditioning: the $\mathrm{R}$ package ggm. J Stat Softw 15(6):1-15

Marsch-Martínez N, Ramos-Cruz D, Irepan Reyes-Olalde J, LozanoSotomayor P, Zúñiga-Mayo VM, De Folter S (2012) The role of cytokinin during Arabidopsis gynoecia and fruit morphogenesis and patterning. Plant J 72(2):222-234

Mei D, Wang H, Hu Q, Li Y, Xu Y, Li Y (2009) QTL analysis on plant height and flowering time in Brassica napus. Plant Breed 128(5):458-465

Merker A, Eriksson D, Bertholdsson N-O (2010) Barley yield increases with undersown Lepidium campestre. Acta Agric Scand Sect B-Soil Plant Sci 60(3):269-273 
Merker A, Nilsson P (1995) Some oil crop properties in wild Barbarea and Lepidium species. Swed J Agric Res 25:173-178

Mohammed H, Akromah R, Abudulai M, Mashark S, Issah A (2014) Genetic analysis of resistance to pod shattering in soybean. J Crop Improv 28(1):17-26

Nilsson P, Johansson SÅ, Merker A (1998) Variation in seed oil composition of species from the genera Barbarea and Lepidium. Acta Agric Scand B-Plant Soil Sci 48(3):159-164

Patterson SE (2001) Cutting loose. Abscission and dehiscence in Arabidopsis. Plant Physiol 126(2):494-500

Pourkheirandish M, Hensel G, Kilian B, Senthil N, Chen G, Sameri M et al. (2015) Evolution of the grain dispersal system in barley. Cell 162(3):527-539

Purugganan MD, Fuller DQ (2009) The nature of selection during plant domestication. Nature 457(7231):843-448

R Core Team (2018) R: a language and environment for statistical computing. R Foundation for Statistical Computing, Vienna, Austria. https://www.R-project.org

Saghai-Maroof MA, Soliman KM, Jorgensen RA, Allard R (1984) Ribosomal DNA spacer-length polymorphisms in barley: mendelian inheritance, chromosomal location, and population dynamics. Proc Natl Acad Sci 81(24):8014-8018

Sato K, Yamane M, Yamaji N, Kanamori H, Tagiri A, Schwerdt JG et al. (2016) Alanine aminotransferase controls seed dormancy in barley. Nat Commun 7:11625

Shastry BS (2002) SNP alleles in human disease and evolution. J Hum Genet 47(11):561-566

Simons KJ, Fellers JP, Trick HN, Zhang Z, Tai Y-S, Gill BS et al. (2006) Molecular characterization of the major wheat domestication gene Q. Genetics 172(1):547-555

Steel RG, Torrie JH (1980) Principles and procedures of statistics: a biometrical approach 2nd edn. McGraw-Hill Book Co., New York, USA

Sugimoto K, Takeuchi Y, Ebana K, Miyao A, Hirochika H, Hara N et al. (2010) Molecular cloning of Sdr4, a regulator involved in seed dormancy and domestication of rice. Proc Natl Acad Sci 107 (13):5792-5797

Sun L, Miao Z, Cai C, Zhang D, Zhao M, Wu Y et al. (2015) GmHs11 , encoding a calcineurin-like protein, controls hard-seededness in soybean. Nat Genet 47(8):939

Tan L, Li X, Liu F, Sun X, Li C, Zhu Z et al. (2008) Control of a key transition from prostrate to erect growth in rice domestication. Nat Genet 40(11):1360

Teng F, Zhai L, Liu R, Bai W, Wang L, Huo D et al. (2013) ZmGA3ox2, a candidate gene for a major QTL, qPH3. 1, for plant height in maize. Plant J 73(3):405-416

Ulén B, Aronsson H (2018) Nitrogen and phosphorus leaching under the potential biennial oilseed plant Lepidium campestre L. in a field trial. Acta Agric Scand, Sect B-Soil Plant Sci 68 (6):555-561

van Gelderen K, van Rongen M, Otten A, Offringa R (2016) An INDEHISCENT-controlled auxin response specifies the separation layer in early Arabidopsis fruit. Mol Plant 9(6):857-869

Van Ooijen J (2006) JoinMap ${ }^{\circledR}$ 4, Software for the calculation of genetic linkage maps in experimental populations. Wageningen 33(10.1371), Kyazma BV

Van Ooijen J (2009) MapQTL 6. Software for the mapping of quantitative trait loci in experimental populations of diploid species. Wageningen, Kyazma BV, Netherlands

Voorrips RE (2002) MapChart: software for the graphical presentation of linkage maps and QTLs. J Hered 93:77-78

Vrebalov J, Pan IL, Arroyo AJM, McQuinn R, Chung M, Poole M et al. (2009) Fleshy fruit expansion and ripening are regulated by the tomato SHATTERPROOF gene. TAGL1 Plant Cell 21 (10):3041-3062

Vukosavljev M, Arens P, Voorrips RE, van't Westende WP, Esselink G, Bourke PM et al. (2016) High-density SNP-based genetic maps for the parents of an outcrossed and a selfed tetraploid garden rose cross, inferred from admixed progeny using the $68 \mathrm{k}$ rose SNP array. Hortic Res 3:16052

Walsh B, Lynch M (2018) Evolution and selection of quantitative traits. Oxford University Press, New York

Wang M, Li W, Fang C, Xu F, Liu Y, Wang Z et al. (2018) Parallel selection on a dormancy gene during domestication of crops from multiple families. Nat Genet 50(10): 1435

Wright LI, Tregenza T, Hosken DJ (2008) Inbreeding, inbreeding depression and extinction. Conserv Genet 9(4):833

Wu W, Liu X, Wang M, Meyer RS, Luo X, Ndjiondjop M-N et al. (2017) A single-nucleotide polymorphism causes smaller grain size and loss of seed shattering during African rice domestication. Nat Plants 3(6): 17064

Wu Y, Zhao S, Li X, Zhang B, Jiang L, Tang Y et al. (2018) Deletions linked to PROG1 gene participate in plant architecture domestication in Asian and African rice. Nat Commun 9 (1):4157

Xu S (2008) Quantitative trait locus mapping can benefit from segregation distortion. Genetics 180(4):2201-2208

Yu B, Lin Z, Li H, Li X, Li J, Wang Y et al. (2007) TAC1, a major quantitative trait locus controlling tiller angle in rice. Plant $\mathrm{J} 52$ (5):891-898

Zeng Z-B (1994) Precision mapping of quantitative trait loci. Genetics 136(4):1457-1468

Zuo J-F, Niu Y, Cheng P, Feng J-Y, Han S-F, Zhang Y-H et al. (2019) Effect of marker segregation distortion on high density linkage map construction and QTL mapping in soybean (Glycine max L.). Heredity 123(5):579-592 